\title{
Microcin-E492-insensitive Mutants of Escherichia coli K12
}

\author{
By ANTHONY P. PUGSLEY, ${ }^{*} \dagger$ FELIPE MORENO AND \\ VICTOR DE LORENZO \\ Unité de Génétique Moléculaire, Institut Pasteur, 25 rue du Dr Roux, Paris 75724, France \\ Unidad de Genética Molecular, Servicio de Microbiología, Hospital Ramón y Cajal, Carretera de \\ Colmenar, Km 9, 1000, Madrid 28034, Spain.
}

(Received 18 April 1986; revised 30 June 1986)

\begin{abstract}
Mutations in three Escherichia coli $\mathrm{K} 12$ genes, $\operatorname{ton} B$, exbB and the newly discovered sem $A$, reduce sensitivity to the low $M_{\mathrm{r}}$ polypeptide antibiotic microcin E492. The products of the ton $B$ and $e x b B$ genes were previously shown to be involved in the uptake of siderophore-complexed iron and in the action of a number of colicins. Strains mutated at or close to semA (collectively referred to as sem mutations) remained fully sensitive to these colicins, and grew as well as wildtype strains under conditions of iron starvation. Expression of a number of sem-lac Z operon fusions was not affected by iron limitation, and sem mutations did not affect the production of iron-regulated outer membrane proteins which are known or thought to be involved in iron uptake. Hfr conjugation and $\mathrm{Pl}$ phage transduction experiments indicated that $\operatorname{sem} A$ is located close to $p a b B$ at 40 min on the $E$. coli $\mathrm{K} 12$ chromosome. This places sem $A$ close to the $m n g$ locus, wherein mutations result in decreased manganese sensitivity. However, strains carrying the sem $A$ mutation exhibited increased manganese sensitivity.
\end{abstract}

\section{INTRODUCTION}

Microcin E492 is a polypeptide antibiotic of $M_{\mathrm{r}} \sim 5000$ produced by Klebsiella pneumoniae and active against Escherichia coli strains, including the laboratory strain K12 (de Lorenzo, 1984). The primary target of microcin E492 seems to be the cytoplasmic membrane, where it causes rapid loss of the transmembrane energy potential (de Lorenzo \& Pugsley, 1985). This implies that microcin E492 crosses the $E$. coli outer membrane.

Colicins, which are generally larger than microcins (Baquero \& Moreno, 1984; Pugsley, 1984), also have to cross the outer membrane in order to reach their targets. The initial stage in this process is attachment to outer membrane receptors, whose production can be blocked by mutations in the appropriate structural genes, thereby causing the cells to become colicin resistant (Pugsley, 1984). Other mutations, such as tonB, tolC and those affecting outer membrane porin production, cause the loss of colicin sensitivity by blocking colicin uptake from the receptor-bound state. These mutants are referred to as colicin tolerant (Pugsley, 1984).

Since microcins are apparently structurally different from most colicins (Baquero \& Moreno, 1984), it is of interest to determine whether they too must adsorb to cell surface receptors in order to cross the outer membrane. These studies are hindered by the very small quantities of the antibiotic which can be prepared in relatively pure form (Baquero \& Moreno, 1984; de Lorenzo, 1984). We have therefore resorted to studies of microcin-insensitive mutants in the hope of identifying cell envelope components involved in microcin uptake. Here we describe the characterization of mutants obtained by selecting for loss of sensitivity to microcin E492.

† Offprint requests to the Paris address.

$\ddagger$ Present address: Department of Biochemistry, University of California, Berkeley, Calif. 94720, USA. 
Table 1. E. coli K12 strains

Strain

Genotype/other characteristics

Source or reference*

PAP488

$\Lambda($ pro-lac) $r p s L$

Laboratory strain

PAP710

semA12::Tn 5 gyrA

PAP1611 seml gyrA $\lambda^{+}$

PAP1612 sem6 gyrA $\lambda^{+}$

PAP1613 sem3 gyrA $\lambda^{+}$

AB3303

thi-l pabB3 hisG4 argE3 lacYI galK2 xyl-5 mtl-1 rpsL700/704

tsx-29/358 supE 44

PAP207 thr leu $\triangle($ proAB-phoE) argE3 trpE hisG4 lacZ galK2 $x y l-5$ mtl-1 rpsL700 gyrA

ZSG113 lacZ83:827 ptsM12 ptsG22 glk-7 rha-4 rpsL223

RC63 gal-55 asnA3 relAl spoTl thi-I hsdR4

PAP1614 ZSG113 sbma12::Tn5 via P1 phage grown on strain PAP710

C600 thr leu fhuA supE44

GUC41 C600 $[\Delta(\operatorname{met} C-e x b B)]$

PAP609 C600 aroB malT: :Tn 5 malPQ: :Tn10

PAP892 AB3303 Mucts ApR

PAP889 araD139 $\triangle($ araCOIBA-leu $) 7697 \Delta$ (proAB-argF-lacIPOZYA)XIII

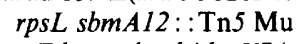

M1967 trpE lys mal gal $\Delta l a c X 74$ thiA rpsL tsx $\lambda^{+}$

BN905 fur : :Tn5 gyrA

BZB1191 gyrA fhuA ::Tn5
Laboratory strain

Bagg \& Neilands (1985)

Laboratory strain
CGSC

Laboratory strain

CGSC

Laboratory strain

* Strains for which no source or reference is given are described in this paper. CGSC, Coli Genetic Stock Center (curator B. Bachmann), Yale University, New Haven, Conn., USA.

\section{METHODS}

Bacterial strains, plasmids and bacteriophages. Strains of $E$. coli $\mathrm{K} 12$ used in this study are listed in Table 1 . The aro $B$ mutation was introduced into various strains by P1 transduction using phage grown on strain PAP609 with selection for kanamycin resistance encoded by $\mathrm{Tn} 5$ inserted in the aroB-linked malT gene. K. pneumoniae strain RYC492 was used as the microcin E492 producer. All other colicin and microcin-producing strains were as listed by Pugsley (1985). Bacteriophage MudX was supplied by C. Gross (University of Wisconsin, Madison, Wis., USA). Plasmids pEG109 (Groisman et al., 1984) and pEG5005 (similar to pEG109 but carrying a kanamycinresistance determinant and the $\mathrm{pBR} 322$ replication origin instead of the chloramphenicol resistance determinant and the pACYC184 replication origin) were supplied by E. Groisman (University of Chicago, Ill., USA). pIP27 is pBR322 carrying functional bla, met $C$ and $e x b B$ genes (I. Saint-Girons \& A. P. Pugsley, unpublished results), and pBJM002 is pACYC177 carrying functional tet and tonB genes (supplied by R. J. Kadner, University of Virginia, Charlottesville, Va., USA).

Media and culture conditions. L broth and L agar (Miller, 1972) were used for most experiments. Where appropriate, antibiotics were used at the following concentrations: kanamycin and chloramphenicol, $25 \mu \mathrm{g} \mathrm{ml}^{-1}$; ampicillin, 200 or $25 \mu \mathrm{g} \mathrm{ml}^{-1}$ (for plasmids and MudX respectively); tetracycline, $15 \mu \mathrm{g} \mathrm{ml}^{-1}$; streptomycin, $100 \mu \mathrm{g} \mathrm{m}^{-1}$; and sodium nalidixate, $50 \mu \mathrm{g} \mathrm{ml}^{-1}$. Minimal medium was M63 (Miller, 1972) containing $0.5 \%$ glucose (all \% values are $w_{j} v$ ) and, where appropriate, amino acids $(0.01 \%)$ and vitamins $(0.007 \%)($ Table 1$)$. Mannose $(0.5 \%)$ replaced glucose in minimal media in tests for $p t s M$ expression. MacConkey $0.5 \%$ mannose agar was also used for these tests. In tests for asparagine utilization, the ammonium sulphate in M63 medium was replaced by $1 \%$ asparagine. Minimal medium was solidified with $1.6 \%$ agar. $100 \mu \mathrm{M}-2,2$-dipyridyl was added to M63 broth cultures to complex iron (Pugsley \& Reeves, 1976). MacConkey 0.5\% lactose medium was used to test the $\mathrm{Lac}^{+}$character of potential MudX-generated Sem mutants. The medium used to test for manganese sensitivity was as described by Silver et al. (1972) $(0.4 \%$ Difco tryptone, $0.25 \% \mathrm{NaCl})$ and was supplemented with $0.2 \%$ glycerol. All cultures were incubated at $37^{\circ} \mathrm{C}$, and broth cultures were well-aerated.

Genetic procedures. Procedures for $\mathrm{P} 1$ phage transduction and for $\mathrm{Hfr}$ conjugation were essentially as described by Miller (1972). Tn 5 insertions were selected by mutagenizing various $E$. coli K12 cultures with $\lambda \operatorname{Tn} 5$ (c1857 rex : :Tn5 029 P80 b2l) and then growing the cultures overnight in $\mathbf{L}$ broth containing kanamycin and $10 \mathrm{~mm}$-sodium citrate. The cells were then plated onto $\mathrm{L}$ agar containing kanamycin and microcin E492. Mutagenesis with MudX was essentially as described by Baker et al. (1983). The mutagenized bacteria derived from strain PAP488 were grown overnight in L broth containing chloramphenicol and ampicillin, and then plated onto MacConkey lactose agar containing the same antibiotics together with microcin E492. The presence of MudX in or close to the $\operatorname{sem} A$ gene in $\mathrm{Lac}^{+}$clones was confirmed by the simultaneous acquisition of kanamycin resistance and loss of chloramphenicol and ampicillin resistance upon transduction by $P 1$ phage grown on a strain 
carrying the semA12: :Tn 5 mutation. All transductants remained insensitive to microcin E492. The sem : : MudX mutations could also be transduced into other bacteria with selection for MudX-encoded chloramphenicol resistance. Mini-Muduction with pEG109 was essentially as described by Groisman et al. (1984). Mixed Mu lysates grown on a microcin-E492-sensitive strain of $E$. coli K12 were used to infect strain PAP892, which was then plated out on minimal medium containing chloramphenicol and thiamin but devoid of $p$-aminobenzoic acid. Colonies appearing after 2-3 d incubation were purified on the same medium. Procedures for the isolation of plasmids by the Holmes and Quigley method, and for transformation, were as described by Maniatis et al. (1982).

General procedures. $\beta$-Galactosidase was assayed according to Miller (1972). Cross streak tests for microcin, colicin and phage sensitivity were essentially as described by Davies \& Reeves (1975 b). Microcin E492 was prepared and assayed as described previously (de Lorenzo, 1984; de Lorenzo \& Pugsley, 1985). Outer membranes were prepared from $5 \mathrm{ml}$ cultures grown to $\mathrm{OD}_{600}=1.0 \mathrm{in} \mathrm{L}$ broth or minimal medium. The cells were harvested, washed once and resuspended in $1 \mathrm{ml} 25 \mathrm{~mm}-\mathrm{Tris} / \mathrm{HCl}(\mathrm{pH} 7.4)$ containing $1 \mathrm{mM}-\mathrm{Mg}^{2+}$, and disrupted by sonication. Cell debris was removed by low-speed centrifugation. Triton X-100 (final concentration $2 \%$ ) was added to the broken cell suspension and the mixture was incubated on ice for $20 \mathrm{~min}$. The outer membrane (Triton-insoluble wall) was then pelleted by centrifugation for $30 \mathrm{~min}$ at $13000 \mathrm{~g}$ in a microcentrifuge. The pellet was drained and resuspended in $100 \mu \mathrm{l}$ sample buffer for SDS polyacrylamide gel electrophoresis (SDS-PAGE) in gels containing $9 \%$ acrylamide with or without $8 \mathrm{M}$-urea. The Tris/glycine buffer system (Pugsley \& Schnaitman, 1979) was used. The gels were stained with Coomassie brilliant blue.

\section{RESULTS}

\section{Isolation of microcin-E492-insensitive mutants}

E. coli $\mathrm{K} 12$ cultures and pooled $\mathrm{Tn} 5$-mutagenized cultures derived therefrom were plated onto $\mathrm{L}$ agar containing varying amounts of microcin $\mathrm{E} 492$ and incubated at $37^{\circ} \mathrm{C}$. Isolated colonies appearing after 1-2 d were purified and tested for sensitivity to microcin E492 and to other microcins, colicins and bacteriophages (Pugsley, 1985). Three classes of mutants were obtained, corresponding to the previously described $\mathrm{TonB}^{-}$and $\mathrm{ExbB}^{-}$phenotypes (Pugsley \& Reeves,

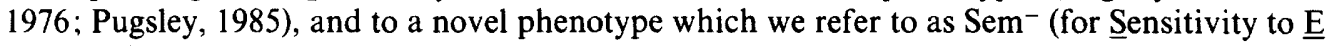
microcin). Unlike the Ton $\mathrm{B}^{-}$and $\mathrm{ExbB}^{-}$mutants, which exhibited extensive cross-resistance to several colicins and, in the case of $\mathrm{TonB}^{-}$, to bacteriophage $\phi 80$ (Pugsley \& Reeves, 1976; Pugsley, 1985), $\mathrm{Sem}^{-}$mutants exhibited reduced sensitivity solely to microcin E492. The Ton $B^{-}$ and $\mathrm{ExbB}^{-}$mutants were insensitive to the highest concentration of microcin available (about 1000 antibiotic units: de Lorenzo, 1984), whereas the $\mathrm{Sem}^{-}$mutants retained a residual level of microcin E492 sensitivity (sensitive to 100-250 antibiotic units; wild-type bacteria were sensitive to 1 antibiotic unit under these assay conditions).

\section{Map location of the sem gene(s)}

In order to confirm that the mutations in strains exhibiting the Ton $\mathrm{B}^{-}$phenotype were indeed located in the ton $B$ gene, P1 phage grown on these strains was used to transduce strain M1967 $(\operatorname{trp} E)$ to $\mathrm{Trp}^{+}$. The $\operatorname{trp} E$ locus is close to $\operatorname{ton} B$. In the three cases studied, $30-40 \%$ of the $\operatorname{Trp}^{+}$ transductants were insensitive to microcin E492. Furthermore, sensitivity to the microcin was recovered upon introduction of $\mathrm{pBJM} 002$, a high-copy-number plasmid carrying the ton $B^{+}$ allele. These results confirmed that the mutations were in ton $B$. Similarly, ExbB- mutants recovered their sensitivity to microcin E492 upon introduction of pIP27, a high-copy-number plasmid carrying the $e x b B^{+}$allele. This result, together with the fact that strain GUC41 $[\triangle($ metC-exbB) $]$ was also microcin-E492-insensitive, whereas the parent strain (C600) was not, confirmed that the $\mathrm{ExbB}^{-}$mutants carried mutations in exbB.

In order to map the mutations conferring the $\mathrm{Sem}^{-}$phenotype, $\mathrm{P} 1$ phage grown on an Semmutant (strain PAP710) derived from a Tn5-mutagenized stock of E. coli K12 was used to introduce the Tn5-generated mutation into a number of $E$. coli $\mathrm{Hfr}$ strains. Loss of sensitivity to microcin E492 was always $100 \%$ cotransduced with resistance to kanamycin. This mutation, henceforth referred to as $\operatorname{sem} A 12:: \mathrm{Tn} 5$, is the prototype of the sem mutations studied here. These strains were then conjugated with strain PAP207 with selection for transfer of Tn5encoded kanamycin resistance and for retention of streptomycin or nalidixate resistance. Transconjugants were checked for loss of auxotrophic or antibiotic resistance markers of strain PAP207. The site of insertion of Tn5 was found to be between $\operatorname{trpE}(28 \mathrm{~min})$ and $g y r A(48 \mathrm{~min})$. 


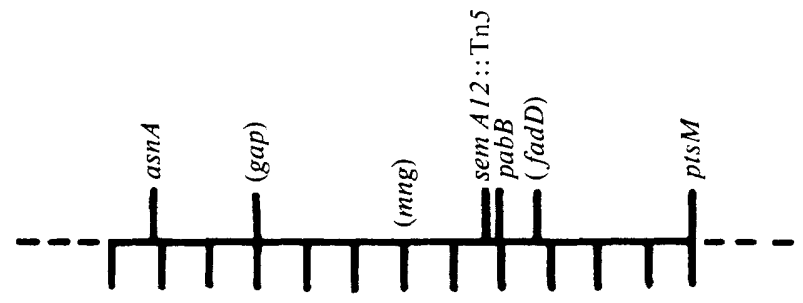

Fig. 1. Partial linkage map of the $40 \mathrm{~min}$ region of the $E$. coli $\mathrm{K} 12$ chromosome redrawn from Bachmann (1983) and showing linkages between genes around semA. Loci in parentheses were not mapped in the present study; their positions are redrawn directly from Bachmann (1983). The precise position of the mng locus is not known.

Table 2. Cotransduction data for sbmA12::Tn5 and adjacent genes and mutations

\begin{tabular}{|c|c|c|c|c|}
\hline $\begin{array}{c}\text { Strain on which the } P 1 \text { phage } \\
\text { was grown }\end{array}$ & Recipient & Selection & $\begin{array}{l}\text { Cotransduced } \\
\text { marker }\end{array}$ & $\begin{array}{c}\text { Percentage } \\
\text { cotransduction }\end{array}$ \\
\hline PAP710 (semA12::Tn5) & AB3303 & $\begin{array}{l}\mathrm{Pab}^{+} \\
\mathrm{Km}^{\mathrm{R}}\end{array}$ & $\begin{array}{l}\mathrm{Km}^{\mathrm{R}} \\
\mathrm{Pab}^{+}\end{array}$ & $\begin{array}{c}98(98 / 100) \\
100(215 / 215)\end{array}$ \\
\hline PAP1611 (seml) & AB3303 & $\mathrm{Pab}^{+}$ & $\mathrm{Sem}^{-}$ & $84(54 / 64)$ \\
\hline PAP1612 (sem6) & AB3303 & $\mathrm{Pab}^{+}$ & $\mathrm{Sem}^{-}$ & $73(62 / 85)$ \\
\hline PAP1613 (sem3) & AB3303 & $\mathrm{Pab}^{+}$ & $\mathrm{Sem}^{-}$ & $86(88 / 97)$ \\
\hline PAP710 (semA12::Tn5) & ZSG113 & $\begin{array}{l}\mathrm{Km}^{\mathrm{R}} \\
\mathrm{Pst}^{+}\end{array}$ & $\begin{array}{l}\text { PtsM }^{+} \\
\text {Sem }^{-}\end{array}$ & $\begin{array}{l}38(38 / 100) \\
33(33 / 100)\end{array}$ \\
\hline PAP710 (semA12:: $\operatorname{Tn} 5)$ & RC63 & $\mathrm{Km}^{\mathrm{R}}$ & $\mathrm{Asn}^{+}$ & $62(62 / 100)$ \\
\hline PAP1614 (semA12::Tn5 ptsM) & AB3303 & $\mathrm{Pab}^{+}$ & $\begin{array}{l}\mathrm{Sem}^{-} \\
\text {PtsM }^{+}\end{array}$ & $\begin{array}{l}96(96 / 100) \\
48(48 / 100)\end{array}$ \\
\hline AB3303 $(p a b B)$ & PAP1614 & PtsM $^{+}$ & $\begin{array}{l}\mathrm{Sem}^{+} \\
\mathrm{Pab}^{-}\end{array}$ & $\begin{array}{l}39(45 / 115) \\
51(51 / 100)\end{array}$ \\
\hline
\end{tabular}

For more precise mapping, $\mathrm{P} 1$ phage grown on strains carrying $\operatorname{sem} A 12:: \operatorname{Tn} 5$ were used to transduce the kanamycin resistance marker into strains carrying mutations between $28 \mathrm{~min}$ and $48 \mathrm{~min}$ on the $E$. coli $\mathrm{K} 12$ chromosome. In this way, we demonstrated that the $\mathrm{Tn} 5$ insertion was linked to $p a b B, p t s M$ and asnA (Table 2), but not to aroD, man-4, lpp-6, pdxH15 or pheS (data not shown). Further, two- and three-point crosses were used to complete the linkage map (Table 2) and to establish the following clockwise gene order on the $E$. coli $\mathrm{K} 12$ chromosome: asnAsemA12::Tn5-pabB-ptsM (Fig. 1).

To determine whether the mutations in other $\mathrm{Sem}^{-}$derivatives mapped at the same site, $\mathrm{P} 1$ phage grown on selected spontaneous mutants were used to transduce strain AB3303 to $\mathrm{Pab}^{+}$. In every case, the mutation was closely linked to $p a b B$, although co-transduction frequencies between these sem mutations and $p a b B$ were not as high as with semA12::Tn5 (Table 2). Two other, independently isolated sem : :Tn 5 mutations were also closely linked to $p a b B$ (data not shown).

\section{Characterization of SemA- mutants}

Because we suspected that the product(s) of the sem gene(s) might function as the microcin E492 receptor, we first looked for changes in outer membrane protein composition in the SemAmutants. Outer membrane protein profiles of wild-type and mutant strains were identical in two different SDS-PAGE systems (Methods). The observation that the products of the exbB and ton $B$ genes are required for microcin E492 sensitivity, and the fact that enterochelin affords low level protection of sensitive cells against the microcin (unpublished observation) suggested that the sem gene product(s), like the receptors for some group B colicins (Davies \& Reeves, $1975 b$; Pugsley \& Reeves, 1976, 1977), might be overproduced during growth under iron-limiting conditions. We therefore used SDS-PAGE to examine outer membrane proteins from parent and mutant bacteria after iron starvation resulting from the presence of an aro $B$ mutation, which prevented production of the siderophore enterochelin, and growth in minimal medium in the 


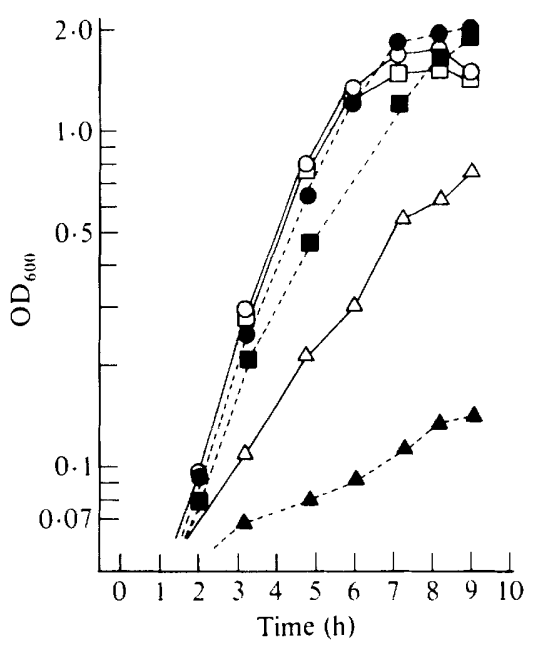

Fig. 2. Effect of manganese on growth of strain PAP710 (semA12::Tn5) (filled symbols and dashed lines) and BZB1 191 ( $/ h u A$ : : Tn5) (this strain, which is isogenic with PAP710, was used in preference to the parent of PAP710 in order to compensate for any effects of $\mathrm{Tn} 5$ on bacterial growth) (open symbols and solid lines). Cells were grown overnight in manganese sensitivity test medium and then diluted $1: 100$ into fresh medium $(\square, \square)$ or fresh medium containing $1.5 \mathrm{mM}^{-\mathrm{MnSO}_{4}}(\triangle, \Delta)$ or $1.5 \mathrm{~mm}$ $\mathrm{MnSO}_{4}+1.5 \mathrm{~mm}-\mathrm{MgSO}_{4}(\mathrm{O}, \mathrm{O})$. Growth was measured as increases in $\mathrm{OD}_{600}$.

presence of the iron chelator 2,2'-dipyridyl (Pugsley \& Reeves, 1976). The parent E. coli K12 strain produced five iron-starvation-induced outer membrane proteins $\left(M_{\mathrm{r}} 70000-84000\right)$, as did all Sem ${ }^{-}$mutants tested (data not shown). We also observed that the $\mathrm{Sem}^{-}$mutants grew as well as the parent strains under iron-limiting conditions (data not shown), which indicated that they do not have a major defect in iron uptake.

In a further attempt to determine whether the sem gene(s) was(were) regulated by the level of iron in the growth medium, we constructed a series of sem-lac $Z$ operon fusions (Methods). In all of the eight independently isolated fusion strains tested, fusion-encoded $\beta$-galactosidase reached 150-200 units $\mathrm{ml}^{-1}$, irrespective of the presence or absence of dipyridyl in the minimal glucose growth medium in the presence of an aro $B$ mutation in the chromosome. Likewise, the level of $\beta$ galactosidase was not affected by the introduction of the fur : :Tn 5 mutation, which is known to derepress iron-regulated genes in E. coli (Bagg \& Neilands, 1985). We therefore concluded that sem is not regulated by the level of free iron in the medium, and does not code for one of the ironregulated outer membrane proteins. We noted, however, that strains carrying presumed semlac $Z$ operon fusions formed darker red colonies (i.e., more strongly $\mathrm{Lac}^{+}$) when plated on MacConkey lactose agar containing dipyridyl.

The $\operatorname{sem} A$ gene is located close to the $m n g$ locus, wherein previously isolated mutations were found to confer increased manganese resistance (Silver et al., 1972; Bachmann, 1983). We therefore tested Sem ${ }^{-}$mutants to see if they exhibited altered sensitivity to manganese. Mutants carrying spontaneous or Tn5-generated sem mutations were more sensitive than parent strains, or other isogenic strains, to $1.5-15 \mathrm{~mm}-\mathrm{MnSO}_{4}$ in liquid culture tests (Fig. 2). The effects of $\mathrm{Mn}^{2+}$ on mutant and wild-type strains were always overcome by including $1.5-15 \mathrm{mM}-\mathrm{Mg}^{2+}$ in the growth medium (Fig. 2). The Sem ${ }^{-}$mutants were also more sensitive to $\mathrm{MnSO}_{4}$ in plate tests (data not shown).

\section{Cloning of $\operatorname{sem} A$}

We made use of the close proximity of the $\operatorname{sem} A$ and $p a b B$ alleles to clone the wild-type sem $A$ allele by selecting for $\mathrm{Pab}^{+}$mini-Muductants of a derivative of strain AB3303 [PAP892 (pabB $\mathrm{Mu} c$ ts $\mathrm{Ap}^{\mathrm{R}}$ ); Methods]. Representatives of six of the eight series of $\mathrm{Pab}^{+}$clones carried plasmids which were able to restore microcin E492 sensitivity to normal, wild-type levels when 
introduced into strain PAP889. This confirmed the genetic linkage between semA and pabB and demonstrated that the wild-type sem $A$ allele was dominant over the mutated allele. The fact that the presence of the recombinant plasmids did not increase microcin sensitivity to levels higher than in wild-type bacteria suggests that the $\operatorname{sem} A$ gene product was not overproduced, or else that it is not normally limiting for microcin E492 sensitivity. However, each cycle of plasmid extraction and retransformation produced a number of clones which did not recover micocin sensitivity. Furthermore, microcin-resistant clones segregated from the microcin-sensitive transformants of strain PAP889 despite continued selection for plasmid-encoded chloramphenicol resistance. The analysis of plasmids derived from these clones indicated that they had sustained deletions which had presumably removed the $\operatorname{sem} A$ gene. Some of the deletions also removed the $p a b B$ gene. We also noted that $\operatorname{sem} A^{+}$plasmids were never obtained among $\mathrm{Pab}^{+}$ mini-Muductants of PAP892 when pEG109 was replaced by pEG5005, which has a higher copy number.

\section{DISCUSSION}

We have adopted a genetic approach for the study of factors which affect the microcin sensitivity of $E$. coli $\mathrm{K} 12$. We have identified two possible candidates for genes encoding hypothetical microcin receptors, the $\operatorname{sem} A$ gene described here and the $\operatorname{sbm} A$ gene affecting sensitivity to microcin B17 (Laviña et al. 1986). Although there are some obvious parallels between the results reported here and those reported previously with microcin B17, there are also some striking differences which may help us to understand how microcins reach their targets. Microcin E492 resembles the group B colicins (Davies \& Reeves, 1975a) in its requirement for $\operatorname{ton} B$ and $e x b B$ gene products, whereas microcin $\mathrm{B} 17$ resembles the group $\mathrm{A}$ colicins (Davies \& Reeves, 1975b), and requires the OmpF porin for efficient killing (Laviña et al., 1986). This almost certainly reflects the use of distinct uptake pathways by the two microcins (Pugsley, 1984), which have different targets [microcin B17 inhibits chromosome replication whereas microcin E492 affects the cytoplasmic membrane energy potential (Herrero \& Moreno, 1986; de Lorenzo \& Pugsley, 1985)].

There is no direct evidence that either the $\operatorname{sim} A$ or the $\operatorname{sem} A$ gene products actually codes for a microcin receptor protein, or indeed that they are involved in translocating the microcin across the outer membrane, although there is indirect evidence for the latter in the case of $s b m A$ (Laviña et al., 1986). sbmA and sem mutations cause specific loss of sensitivity to a single lethal agent, which is also sometimes the case with mutations which affect colicin receptors (Pugsley, 1984). However, unlike $s b m A$ and most of the mutations preventing synthesis of colicin receptors, sem mutations do not cause the complete loss of microcin sensitivity. This may indicate that there are two pathways for microcin uptake. Only TonB- mutations were obtained by selecting for high-level microcin E492 resistance in strains carrying an sem mutation.

Studies with operon fusions indicated that both $\operatorname{sim} A$ and $\operatorname{sem} A$ genes are poorly expressed (this study and M. Laviña \& F. Moreno, unpublished data; see also Laviña et al., 1986), but $s b m A^{+}$can be stably maintained in high-copy-number plasmids (Laviña et al., 1986) whereas $\operatorname{sem} A^{+}$(or a closely linked gene) cannot. SbmA $A^{-}$mutants do not have any recognizable phenotype other than their loss of sensitivity to microcin B17, whereas Sem- mutants are more sensitive to manganese than parent strains. This latter phenomenon may be useful in further studies into the mechanism of microcin E492 uptake and the physiological role of the sem gene product(s). For example, it may be that a sem gene codes for a manganese efflux pump (Silver $e t$ al., 1972). This pump may have been more efficient or present in increased amounts in the Mngmutants, whereas it may be completely absent from Sem ${ }^{-}$mutants. Alternatively, sem mutations may exert a polar effect on the expression of another gene whose product is involved in manganese transport. The results presented here do not completely exclude a possible interaction between iron transport and microcin E492 action. The evidence that the ton $B$ and $e x b B$ gene products are required for microcin E492 action, and the fact that enterochelin affords low-level protection against this microcin, are both indicative of such an interaction. Enterochelin may protect against microcin E492 in a manner similar to that by which it protects against colicins $\mathrm{G}, \mathrm{H}, \mathrm{Ia}, \mathrm{Ib}$ and $\mathrm{V}$, rather than by preventing the interaction of microcin $\mathrm{E} 492$ 
with a cognate receptor, as is the case for colicins B and D (Pugsley \& Reeves, 1976). The absence of any indication of iron regulation of semA regulation except on MacConkey lactose agar may indicate a more complex interaction between microcin E492 uptake and ion transport than is the case for other TonB-dependent agents.

We are grateful to Maxime Schwartz for his interest in this work. Financial support was provided by the Centre National de la Recherche Scientifique (UA 041149 and ATP CP96), by the Ministère de la Recherche et de la

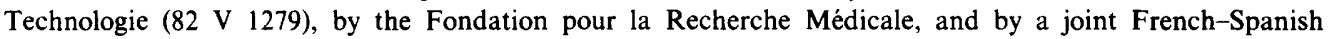
government scientific exchange program. V.deL. was the recipient of an EMBO short-term fellowship.

\section{REFERENCES}

BachmanN, B. J. (1983). Linkage map of Escherichia coli K-12. Edition 7. Microbiological Reviews 47, 180230.

BagG, A. \& Neilands, J. B. (1985). Mapping of a mutation affecting regulation of iron uptake systems in Escherichia coli K-12. Journal of Bacteriology 161, 450-453.

BaKer, T. A., Howe, M. M. \& Gross, C. A. (1983). MudX, a derivative of MudI (lac Ap ${ }^{R}$ ) which makes stable lac $Z$ fusions at high temperature. Journal of Bacteriology 156, 970-974.

BAquero, F. \& MoReno, F. (1984). The microcins. FEMS Microbiology Letters 23, 117-124.

DAVIES, J. K. \& ReEves, P. (1975a). Genetics of resistance to colicins in Escherichia coli K-12: crossresistance among colicins of group B. Journal of Bacteriology 123, 96-101.

Davies, J. K. \& Reeves, P. (1975b). Genetics of resistance to colicins in Escherichia coli K-12: cross resistance among colicins of group A. Journal of Bacteriology 123, 102-117.

Groisman, E. A., Castillho, B. A. \& Casadaban, M. J. (1984). In vivo cloning and adjacent gene fusing with a mini-Mu-lac bacteriophage containing a plasmid replicon. Proceedings of the National Academy of Sciences of the United States of America 81, 14801483.

Herrero, M. \& Moreno, F. (1986). Microcin B17 blocks DNA replication and induces the SOS system in Escherichia coli. Journal of General Microbiology 132, 393-402.

Laviña, M., Pugsley, A. P. \& Moreno, F. (1986). Identification, mapping, cloning and characterization of a gene $(s b m A)$ required for microcin $\mathrm{B} 17$ action on Escherichia coli K12. Journal of General Microbiology 132, 1685-1693.

DE LORENZO, V. (1984). Isolation and characterization of microcin E492 from Klebsiella pneumoniae. Archives of Microbiology 139, 72-75.

de Lorenzo, V. \& Pugsley, A. P. (1985). Microcin E492, a low molecular weight peptide antibiotic which causes depolarization of the Escherichia coli cytoplasmic membrane. Antimicrobial Agents and Chemotherapy 25, 666-669.

Maniatis, T., Fritsch, E. F. \& Sambrook, J. (1982). Molecular Cloning: A Laboratory Manual. Cold Spring Harbor, NY: Cold Spring Harbor Laboratory.

MilleR, J. H. (1972). Experiments in Molecular Genetics. Cold Spring Harbor, NY: Cold Spring Harbor Laboratory.

Pugsley, A. P. (1984). The ins and outs of colicins. Microbiological Sciences 1, 168-175, 203-205.

PugsLeY, A. P. (1985). Escherichia coli K12 strains for use in the identification and characterization of colicins. Journal of General Microbiology 131, 369376.

Pugsley, A. P. \& Reeves, P. (1976). Characterization of group B colicin-resistant mutants of Escherichia coli K-12: colicin resistance and the role of enterochelin. Journal of Bacteriology 127, 218-228.

PugsLey, A. P. \& ReEves, P. (1977). The role of colicin receptors in the uptake of ferrienterochelin by Escherichia coli K-12. Biophysical and Biochemical Research Communications 74, 903-911.

Pugsley, A. P. \& Schnaitman, C. A. (1979). Factors affecting the electrophoretic mobility of the major outer membrane proteins of Escherichia coli in polyacrylamide gels. Biochimica et biophysica acta $581,163-178$.

Silver, S., Johnseine, P., Whitney, E. \& Clark, D. (1972). Manganese-resistant mutants of Escherichia coli: physiological and genetic studies. Journal of Bacteriology 110, 186-195. 